\title{
Adenocarcinoma de páncreas: actualizaciones terapéuticas
}

\author{
P. KHOSRAVI SHAHI, V. M. DÍAZ MUÑOZ DE LA ESPADA \\ Servicio de Oncología Médica. Hospital General Universitario Gregorio Marañón. \\ Madrid
}

\author{
PANCREATIC CANCER: THERAPEUTICAL UPDATE
}

\begin{abstract}
RESUMEN
El cáncer de páncreas exocrino continúa siendo un gran problema de salud sin resolver. Debido a las dificultades en el diagnóstico, la agresividad de la enfermedad, y la ausencia de un tratamiento sistémico efectivo, generalmente menos del $5 \%$ de los pacientes con cáncer de páncreas sobreviven a los 5 años tras el diagnóstico. Por ello, la tasa de incidencia y de mortalidad son prácticamente iguales. La supervivencia mediana en cáncer de páncreas metastásico es aproximadamente de unos 6 meses. La cirugía continúa siendo la única opción terapéutica potencialmente curativa, y el tratamiento de referencia en los pacientes con enfermedad diseminada es la gemcitabina. Nuevas estrategias están en investigación tanto para la enfermedad resecable como irresecable, tales como la quimiorradioterapia adyuvante o neoadyuvante o las combinaciones de gemcitabina con nuevos agentes citostáticos (oxaliplatino, cetuximab, gefitinib, bevacizumab) con resultados esperanzadores. En pacientes con enfermedad localmente avanzada y buen estado general, la quimiorradioterapia concomitante debe ser tenida en cuenta.
\end{abstract}

PALABRAS CLAVE: Cáncer de páncreas. Gemcitabina. K-ras. GEMOX. Carcinomatosis peritoneal.

\begin{abstract}
Cancer of the exocrine pancreas continues to be a major unsolved health problem. Because of difficulties in diagnosis, the aggressiveness of pancreatic cancers, and the lack of effective systemic therapies, generally fewer than $5 \%$ of patients with adenocarcinoma of the pancreas survive 5 years after diagnosis. Thus, incidence rates and mortality rates are virtually identical. The median survival in metastatic pancreatic cancer is nearly six months.Today, surgery remains the only curative therapeutic option, and the standard treatment in patients with advanced disease is gemcitabine.New strategies for resectable and unresectable pancreatic cancer are under active investigation, such as neoadjuvant or adjuvant chemoradiothearapy or combinations of gemcitabine with new cytotoxic agents (oxaliplatin, cetuximab, gefitinib, bevacizumab) with promising results. In patients with locally advanced pancreatic cancer and good performance status, chemoradiotherpy should be considered.
\end{abstract}

KEY WORDS: Pancreatic cancer. Gemcitabine. K-ras, GEMOX. Peritoneal metastases.

Khosravi Shahi P, Díaz Muñoz de la Espada VM. Adenocarcinoma de páncreas: actualizaciones terapéuticas. An Med Interna (Madrid) 2005; 22: 390-394.

\section{INTRODUCCIÓN}

El adenocarcinoma es la neoplasia maligna más frecuente de páncreas, con un pronóstico infausto debido a que la gran mayoría de los casos se presentan en estadios avanzados (1), por lo que no son candidatos a la cirugía, siendo esta la única modalidad potencialmente curativa (2-4). En España, el cáncer de páncreas es la tercera neoplasia más frecuente del tubo digestivo, y su incidencia en los países occidentales es de 10 por 100.000. Es la cuarta causa de muerte por cáncer en los países occidentales.
El 90\% de las neoplasias del páncreas exocrino son adenocarcinomas (Tabla I). El 70\% de los adenocarcinomas de páncreas se localizan a nivel de cabeza del páncreas, y el 30\% restantes a nivel de cuerpo y cola. Estos últimos generalmente se presentan en estadios avanzados con presencia de metástasis hepáticas o carcinomatosis peritoneal. La supervivencia mediana sin tratamiento es de unos 4-6 meses, y con gemcitabina la supervivencia al año es de $20-25 \%$.

El 10\% restante está formado por los tumores quísticos de páncreas, tumores neuroendocrinos enteropancreáticos, linfomas y metástasis pancreáticas de otras neoplasias.

Trabajo aceptado: 27 de marzo de 2005 


\section{TABLA I}

VARIANTES HISTOLÓGICAS DEL CÁNCER DE PÁNCREAS EXOCRINO

1. Adenocarcinoma pancreático $(90 \%)$

2. Tumores pancreáticos quísticos: -Cistoadenocarcinoma mucinoso

- Cistoadenocarcinoma seroso

- Tumor pseudopapilar sólido

3. Tumores neuroendocrinos enteropancreáticos

4. Linfomas

5. Metástasis de otros tumores sólidos:

-Cáncer de mama; cáncer de pulmón y melanomas
TABLA II

FACTORES DE RIESGO DEL ADENOCARCINOMA DE PÁNCREAS

1. Antecedentes familiares de cáncer de páncreas

2. Edad avanzada

3. Tabaquismo

4. Exposición ocupacional (Industria petroquímica)

5. Obesidad

6. Pancreatitis crónica (especialmente las hereditarias)

7. Dieta rica en carnes y pescados fritos; y pobre en frutas y verduras

8. Gastrectomía parcial

9. Lesiones premalignas (en especial el cistoadenoma mucinoso)

10. Alteraciones genéticas/hereditarias (mutación K-ras, APC, DCC, P-53)

\section{ETIOPATOGENIA}

La etiología del cáncer pancreático es desconocida. Se sabe que en la mayoría de los adenocarcinomas de páncreas existen alteraciones genéticas y/o cromosómicas. En el $70 \%$ de los casos se halla mutaciones del oncogén K-ras, lo que resulta interesante en el diagnóstico de la enfermedad mediante su detección en el jugo pancreático o aspirado de masas pancreáticas por punción con aguja fina. Otras alteraciones genéticas son deleciones o mutaciones del gen supresor p53, APC o DCC. Entre las alteraciones cromosómicas destacan las deleciones del 9p, 17p y 18q, que contienen muchos genes supresores.

Los factores de riesgo para el adenocarcinoma de páncreas incluyen (Tabla II):

1. Antecedentes familiares de cáncer de páncreas: $5-10 \%$ de los cánceres pancreáticos son hereditarios (5).

2. Edad avanzada (> 60 años): el $80 \%$ de los casos se producen entre los 60 y los 80 años de edad, siendo la edad media al diagnóstico de unos 65 años. El cáncer de páncreas es raro en personas menores de 45 años.

3. Tabaquismo: el efecto nocivo del tabaco se debe a las aminas aromáticas presentes en le humo de tabaco (6).

4. Sexo: el cáncer de páncreas es más frecuente en los varones, sin embargo en los no fumadores no hay predominio sexual.

5. Exposición ocupacional: es más frecuente entre los trabajadores de la industria petroquímica, en relación con una mayor exposición a las aminas aromáticas.

6. Obesidad: parece aumentar el riesgo de cáncer de páncreas (7).

7. Dieta rica en grasas, pescado y carnes fritas, y pobre en frutas y vegetales (8).

8. Pancreatitis crónica: la pancreatitis crónica aumenta el riesgo de padecer cáncer de páncreas con un riesgo acumulado de $4 \%$ a los 20 años. Las pancreatitis crónicas hereditarias tienen un mayor riesgo de malignización (9).

9. El consumo de café y alcohol no son factores de riesgo de cáncer de páncreas (10).

10. Lesiones premalignas: el cistoadenoma mucinoso es el que tiene un mayor riesgo de malignización.

11. Gastrectomía parcial: puede aumentar el riesgo de cáncer de páncreas a los 15-20 años.

\section{MANIFESTACIONES CLÍNICAS}

El cáncer de páncreas suele ser asintomático en las fases iniciales de la enfermedad, o bien se presenta con síntomas inespecíficos. Entre las manifestaciones clínicas cabe destacar el síndrome constitucional (pérdida de peso llamativa, astenia y anorexia) y caquexia, ictericia mucoconjuntival, dolor abdominal, diarrea con esteatorrea, diabetes mellitus, náuseas, vómitos, tromboflebitis migratoria y hemorragia digestiva (11).

El dolor abdominal es el síntoma más frecuente, suele tratarse de un dolor epigástrico y/o en ambos hipocondrios, de intensidad severa, que se irradia "en cinturón" a las regiones dorsolumbares. Suele tener un predominio nocturno, impide conciliar el sueño o despierta al paciente, empeora con las comidas y con el decúbito supino, y se alivia con la flexión ventral de la columna vertebral. El dolor se debe a dos motivos: a) invasión perineural del plexo celíaco y compresión de las estructuras vecinas; b) obstrucción parcial o total del conducto pancreático. La ictericia se debe en el caso de los tumores de cabeza de páncreas a una obstrucción de la vía biliar, y en los casos de tumores de cuerpo y cola a la presencia de metástasis hepáticas.

En la exploración física se puede poner de manifiesto la presencia de ictericia, lesiones por rascado, palpación de una vesícula distendida (signo de Courvoisier), a veces se palpa una masa abdominal, ascitis por carcinomatosis peritoneal o menos frecuentemente por hipertensión portal.

\section{DIAGNÓSTICO}

Ante la sospecha clínica de cáncer de páncreas se debe recurrir a una técnica de imagen, siendo la Tomografía Computarizada (TC) de alta resolución con contraste intravenoso la mejor prueba inicial, aunque algunos autores proponen la ecografía abdominal como la primera prueba a realizar.

La TC permite al mismo tiempo evaluar la localización y tamaño del tumor, la extensión local y a distancia del tumor. La resonancia magnética no mejora la información aportada por la TC. 
Otras técnicas a tener el cuenta en el diagnóstico del adenocarcinoma de páncreas son la CPRE y la ecoendoscopia, estas dos técnicas además permiten obtener muestra para estudio citológico y genético (mutación de K-ras). El diagnóstico de cáncer de páncreas requiere la confirmación histológica o citológica, a través de las muestras obtenidas por punción aspiración con aguja fina dirigida por ecografía o TC o ecoendoscopia; o bien por cepillado/aspiración del jugo pancreático mediante CPRE.

El marcador tumoral CA 19.9, que es sintetizado por el epitelio del árbol biliar y páncreas exocrino, suele ser un buen indicador de enfermedad diseminada cuando se encuentra cifras muy elevadas.

\section{ACTITUD TERAPÉUTICA}

La única modalidad terapéutica potencialmente curativa en el cáncer de páncreas es la cirugía. Pero, la mayoría de los casos son irresecables. Tan sólo el $20 \%$ de los cánceres de páncreas son candidatos a la cirugía (12).

La resecabilidad del tumor viene determinado por la ausencia de metástasis a distancia y la ausencia de afectación de las estructuras vasculares vecinas. Para los tumores de cabeza de páncreas la técnica quirúrgica de elección es la duodenopancreatectomía cefálica de Whipple; mientras que para los tumores de cuerpo y cola la técnica de elección es la pancreatectomía distal o total con o sin esplenectomía.

Sin embargo, el $80 \%$ de los tumores de páncreas son irresecables (40\% son localmente avanzados y $40 \%$ presentan metástasis), en estos casos las posibilidades terapéuticas tienen una intención paliativa.

$\mathrm{Si}$ existe una ictericia obstructiva (colestasis) por compresión de la vía biliar se puede recurrir a diversas técnicas de drenaje de la vía biliar:

1. Técnicas conservadoras: drenaje biliar interno y/o externo mediante la colocación de stent y tubos de drenaje, mediante CPRE y/o técnicas de radiología intervensionista (CPTH).

2. Técnicas quirúrgicas: la técnica de elección es la colecistoyeyunostomía, y si esta no es posible entonces la coledocoyeyunostomía.

En caso de obstrucción o estenosis gastroduodenal por extensión local del tumor, se puede colocar un stent duodenal o bien realizar una gastroyeyunostomía (13).

El tratamiento paliativo debe incluir siempre un buen control del dolor mediante el empleo escalonado de los analgésicos según la escala de la Organización Mundial de la Salud, y evitar los cuadros malabsortivos mediante la administración de suplementos enzimáticos. Si el dolor es medicamente intratable se puede recurrir al bloqueo neurolítico celíaco o esplácnico, siendo su principal complicación la hipotensión postural $(14,15)$.

\section{ADENOCARCINOMA DE PÁNCREAS RESECABLE}

Sólo una minoría se puede beneficiar de la cirugía con intención curativa ( adenocarcinomas menores de $6 \mathrm{~cm}$ en su diámetro mayor, confinados al páncreas, sin metástasis, ni invasión de estructuras vasculares), siendo las técnicas de elección la dudenopancreatectomía cefálica de Whipple (en los tumores de cabeza) y pancreatectomía distal (en los tumores de cuerpo y cola).

Debido al alto riesgo de recidiva local y a distancia a pesar de la cirugía radical, se considera oportuno intentar añadir al tratamiento quirúrgico, un tratamiento adyuvante, en forma de quimioterapia o quimioradioterapia $(13,16)$.

Las diversas posibilidades de tratamiento adyuvante son:

1. Quimiorradioterapia neoadyuvante/ preoperatoria:

—Radioterapia de 45-50,4 Gy asociada a tegafur oral, seguido de radioterapia intraoperatoria (RIO) (17). Se ha estando aplicando con resultados esperanzadores en nuestro centro. Consiste en la asociación de radioterapia externa de 45-50,4 Gy, dividido en 1,8 Gy/fracción, con tegafur 400 $\mathrm{mg} / 8$ horas via oral de manera continua mientras dura el periodo de la radioterapia, incluyendo fines de semana, vacaciones o períodos en los que se interrumpe momentáneamente la radioterapia. Tras completar la quimiorradioterapia concomitante se realiza la resección del tumor con administración de RIO de 10-15 Gy.

-Otra variante es la asociación de radioterapia con quimioterapia basada en 5-Fluorouracilo(5FU $300 \mathrm{mg} / \mathrm{m}^{2} /$ día por 5 días a la semana) desarrollada por Spitz y cols. (18).

-Otro esquema de quimiorradioterapia concomitante se basa en la administración de gemcitabina, en el seno de un ensayo clínico que se encuentra en fase II, con resultados esperanzadores.

2. Quimiorradioterapia coadyuvante/ postoperatoria frente a quimioterapia postoperatoria:

El estudio ESPAC-1, que ha sido severamente criticado, es la serie más larga de la que disponemos (19). Los pacientes fueron tratados con cirugía seguida de quimioterapia sola, quimiorradioterapia concomitante o quimiorradioterapia concomitante seguida de quimioterapia (con esquemas basados en 5FU). Dicho estudio evidenció que la quimiorradioterapia concomitante postcirugía no ofrece beneficios, en comparación con la quimioterapia coadyuvante sola. Los autores recomiendan la quimioterapia con esquema $5 \mathrm{FU} 420 \mathrm{mg} / \mathrm{m}^{2} /$ día en bolus intravenoso durante 5 días, asociado a leucovorin 20 $\mathrm{mg} / \mathrm{m}^{2} /$ día en bolus intravenoso durante 5 días con una periodicidad de 28 días, con un número total de 6 ciclos.

En el estudio EORTC se está ensayando una quimiorradiación postoperatoria basada en gemcitabina, consistente en 2 ciclos de gemcitabina, seguido por gemcitabina semanal por 5 semanas concomitante con radioterapia de 50,4 Gy.

\section{ADENOCARCINOMA DE PÁNCREAS LOCALMENTE AVANZADO (IRRESECABLE)}

El $40 \%$ de los casos se diagnostican en esta fase, y el tratamiento depende del estado general del paciente y de la extensión del tumor. Así, en los pacientes con buen estado general y ausencia de carcinomatosis peritoneal descartada mediante técnicas de imagen (resonancia magnética o tomografía computerizada) y/o por laparoscopia o laparotomía se debe intentar la quimioirradiación concomitante (12); con la posibilidad de conseguir en algunos pocos casos una reducción de la masa tumoral, pasando a ser una enfermedad resecable (20).

En el resto de los casos de adenocarcinoma de páncreas localmente avanzado hay que recurrir a la quimioterapia sola con esquemas basados en gemcitabina. 


\section{ADENOCARCINOMA DE PÁNCREAS AVANZADO (METÁSTASICO)}

El uso de la quimioterapia en los casos de adenocarcinoma de páncreas avanzado ha demostrado un beneficio en la supervivencia y en la calidad de vida de los pacientes en comparación con el tratamiento paliativo (21-25). El agente quimioterápico más activo en el cáncer de páncreas diseminado es la gemcitabina, siendo el primer agente que ha demostrado una mejoría de los síntomas relacionados con el cáncer de páncreas (reducción del dolor y de la necesidad de analgesia) y de la supervivencia (26-30).

Por tanto, en el tratamiento del cáncer de páncreas avanzado se debe emplear esquemas de tratamiento basados en gemcitabina, ya sea en monoterapia o bien en combinación con otros agentes. Actualmente existe un estudio en fase II, que viene a demostrar la superioridad de la perfusión a dosis fija de gemcitabina en monoterapia con respecto al esquema convencional (31). La perfusión a dosis fija de gemcitabina consiste en la administración de gemcitabina a una velocidad de $10 \mathrm{mg} / \mathrm{m}^{2} /$ minuto ( $1.500 \mathrm{mg} / \mathrm{m}^{2}$ en 150 minutos) los días 1,8 y 15 de cada ciclo de 28 días.

En cuanto a las poliquimioterapias basadas en gemcitabina cabe mencionar que la asociación de 5-FU o de irinotecan a la gemcitabina no aporta ninguna ventaja (32-34). Una asociación con resultadores esperanzadores en cuanto a la tasa de respuesta y supervivencia libre de enfermedad se refiere, es el denominado GEMOX (gemcitabina y oxaliplatino), sin embargo aun no hay datos sobre la supervivencia global (35). Otras asociaciones que se están investigando son la gemcitabina con bevacizumab (36), gemcitabina con gefitinib o cetuximab (37), y gemcitabina con cisplatino.

\section{Bibliografía}

1. Lowenfels A, Maisonneuve P. Pancreatic cancer: development of a unifying etiologic concept.Ann NY Acad Sci 1999; 880: 191-200.

2. Warshaw AL, Fernández-del-Castillo C. Pancreatic carcinoma. N Engl J Med 1992; 326: 455-465.

3. Rosewicz S, Wiedenmann B. Pancreatic cancer. Lancet 1997; 349: 485489.

4. Brand R. The diagnosis of pancreatic cancer. Cancer J 2001; 7: 287297.

5. Lynch HT, Fitzsimmons ML, Smyrk TC, Lanspa SJ, Watson P, McClellan J, et al. Familial pancreatic cancer: clinicopathological study of 18 nuclear families. Am J Gastroenterol 1990; 85: 54-60.

6. Gold EB, Goldin SB. Epidemiology of and risk factors for pancreatic cancer. Surg Oncol Clin N Am 1998; 7: 67-91.

7. Wolk A, Gridley G, Svensson M, Nyren O, McLaughlin JK, Fraumeni JF. A prospective study of obesity and cancer risk (Sweden). Cancer Causes Control 2001; 12: 13-21.

8. Michaud DS, Giovannucci E, Willet WC, Colditz GA, Fuchs CS. Coffee and alcohol consumption and the risk of pancreatic cancer in two prospective United States cohorts. Cancer Epidemiol Biomarkers Prev 2001; 10: 429-437.

9. Lowenfels AB, Maisonneuve P, DiMagno EP, Elitsur Y, Gastes LK Jr, Perrault $\mathrm{J}$, et al. International Hereditary Pancreatitis Study Group: Hereditary pancreatitis and the risk of pancreatic cancer. J Natl Cancer Inst 1997; 89: 442-446.

10. Baghurst PA, McMichael AJ, Slavotinek AH, Baghurst KI, Boyle P, Walker AM. A case-control study of diet and cancer of the pancreas. Am J Epidemiol 1991; 134: 167-179.

11. Ballinger AB, McHugh M, Catnach SM, Alstead EM, Clark ML. Symptom relief and quality of life after stenting for malignant bile duct obstruction. Gut 1994; 35: 467-470.

12. Cutsem EV, Aerts R,Haustermans K,Topal B, Steenbergen WV, Verslype C.Systemic treatment of pancreatic cancer.Eur J Gastroenterol Hepatol 2004; 16: 265-274

13. Carr-Locke DL. Role of endoscopic stenting in the duodenum. Ann Oncol 1999; 10: S261-S264.

14. Rykowski JJ, Hilgier M. Efficacy of neurolytic celiac plexus block in varying locations of pancreatic cancer: influence on pain relief. Anesthesiology 2000; 92: 347-354.

15. Gunaratnam NT, Sarma AV, Norton ID, Wiersema MJ. A prospective study of EUS-guided celiac plexus neurolysis for pancreatic cancer pain. Gastrointest Endosc 2001; 54: 316-324.

16. Metha V, Fisher G, Ford J. Adjuvant radiotherapy and concomitant 5fluorouracil by protracted venous infusion for resected pancreatic cancer.Int J Radiat Oncol Biol Phys 2000; 48: 1483-7.
17. Calvo FA, Matute R, García-Sabrido JL, Gomez-Espí M, Martínez NE, Lozano MA, et al. Neoadjuvant chemorradiation with tegafur in cancer of páncreas. Initial Analysis of clinical tolerance and outcome. Am J Clin Oncol 2004; 27: 343-349.

18. Spitz F, Abbruzzese J, Lee J. Preoperative and postoperative chemoradiation strategies in patients treated with pancreaticodudenectomy for adenocarcinoma of pancreas.J Clin Oncol 1997; 15: 928-37.

19. Neoptolemos JP, Stocken DD, Friess H, Bassi C,Dunn JA, Hickey H, et al. A Randomized Trial of Chemoradiotherapy and Chemotherapy after Resection of Pancreatic Cancer. N Engl J Med 2004; 350: 1200-10.

20. Bajetta E, Di-Bartolomeo M, Stani SC, Artale S, Ricci SB, Bozzetti F, et al. Chemoradiotherapy as preoperative treatment in locally advanced unresectable pancreatic cancer patients: results of a feasibility study. Int J Radiat Oncol Biol Phys 1999; 45: 285-289.

21. Mallinson CN, Rake MO, Cocking JB, Fox CA, Cwynarski MT, Diffey BL, et al. Chemotherapy in pancreatic cancer: results of a controlled, prospective, randomised, multicentre trial. Br Med J 1980; 281: 15891591.

22. Frey C, Twomey P, Keehn R, Elliott D, Higgings G. Randomized study of 5-FU and CCNU in pancreatic cancer: report of the Veterans Administration Surgical Adjuvant Cancer Chemotherapy Study Group. Cancer 1981; 47: 27-31.

23. Andersen JR, Friis-Moller A, Hancke S, Roder O, Steen J, Baden H. A controlled trial of combination chemotherapy with 5-FU and BCNU in pancreatic cancer. Scand J Gastroenterol 1981; 16: 973-975.

24. Palmer KR, Kerr M, Knowles G, Cull A, Carter DC, Leonard RC. Chemotherapy prolongs survival in inoperable pancreatic carcinoma. Br J Surg 1994; 81:882-885.

25. Glimelius B, Hoffman K, Sjoden PO, Jacobsson G, Sellstrom H, Enander LK, et al. Chemotherapy improves survival and quality of life in advanced pancreatic and biliary cancer. Ann Oncol 1996; 7: 593-600.

26. Abbruzzese J. Phase I studies with the novel nucleoside analog gemcitabine. Sem Oncol 1996; 23: 25-31.

27. Storniolo AM, Enas NH, Brown CA, Voi M, Rothenberg ML, Schilsky R. An investigational new drug treatment program for patients with gemcitabine. Results for over 3000 patients with pancreatic carcinoma. Cancer 1990; 85: 1261-1268.

28. Burris HA, Moore MJ, Anderson J, Green MR, Rothenberg ML, Modiano MR, et al. Improvements in survival and clinical benefit with gemcitabine as first-line therapy for patients with advanced pancreas cancer: a randomized trial. J Clin Oncol 1997; 15: 2403-2413.

29. Van Riel JM, van Groeningen CJ, Pinedo HM, Giaccone G. Current chemotherapeutic possibilities in pancreaticobiliary cancer. Ann Oncol 1999; 10 (Supl. 4): S157-S161. 
30. Van Cutsem E, Haustermans K, Van Steenbergen W. New treatment possibilities for pancreatic and biliary tumours. Ann Oncol 2000; 11: 165-169.

31. Tempero M, Plunket W, Ruiz van Haperen V, Hainsworth J, Hochster $\mathrm{H}$, Lenzi R, et al. Randomized phase II comparison of dose-intense gemcitabine: thirty-minute infusion and fixed dose rate infusion in patients with pancreatic adenocarcinoma. J Clin Oncol 2003; 21: 34023408.

32. Di Constanza F, Sdrobollini A, Carlini P, Massida R, Mattioli R, Ioq A, et al. Gemcitabine alone or in combination with 5-FU continuous infusion in the treatment of advanced pancreatic cancer: a GOIRC randomized phase II trial. Proc Am Soc Clin Oncol 2001; 20:154a.

33. Berlin J, Catalano P, Thomas J, Kugler J, Haller D, Benson A. Phase III study of gemcitabine in combination with fluorouracil versus gemcitabine alone in patients with advanced pancreatic carcinoma: Eastern Cooperative Oncology Group Trial E2297. J Clin Oncol 2002; 20: 3270-3275.

34. Rocha Lima CMS, Rotche R, Jeffery M, Trudeau M, Cisar LA, Mor- ganti A, et al. A randomized phase 3 study comparing efficacy and safety of gemcitabine (GEM) and irinotecan (I), to GEM alone in patients (pts) with locally advanced or metastatic pancreatic cancer who have not received prior systemic therapy [Abstract]. Proc Am Soc Clin Oncol 2003; 22:251 (1005).

35. Louvet C, Labianca R, Hammel P, Lledo G, De Braud F, Andre T, et al. Gemcitabine versus GEMOX (gemcitabine + oxaliplatin) in non respectable pancreatic adenocarcinoma: interim results of the GERCOR/GISCAD Intergroup Phase III [Abstract]. Proc Am Soc Clin Oncol 2003; 22: 250 (1004)

36. Kindler HL, Ansari R, Lester E, Locker G, Nattam S, Stadler WM, et al. Bevacizumab (B) plus gemcitabine (G) in patients (pts) with advancedpancreatic cancer (PC) [Abstract]. Proc Am Soc Clin Oncol 2003; 22: 259 (1037)

37. Abbruzesse J, Rosenberg A, Xiang Q, Lo Buglio A, Schmidt W, Wolff $\mathrm{R}$, et al. Phase II study of antiepidermal growth factor receptor (EGFR) antibody cetuximab (IMC-C225) in combination with gemcitabine in patients with advanced pancreatic cancer [Abstract]. Proc Am Soc Clin Oncol 2001; 20: 130 (518). 